\title{
TIME COMPRESSION OF X-RAY FREE-ELECTRON LASER PULSES UNDER CONDITIONS OF BRAGG DIFFRACTION
}

\author{
Vladimir A. Bushuev \\ Lomonosov Moscow State University, Faculty of Physics. http://www.phys.msu.ru \\ 119991 Moscow, Russian Federation \\ vabushuev@yandex.ru
}

Abstract. In the last years several laboratories actively work on construction of $\mathbf{X}$-ray free electron lasers (XFEL) with wavelength of radiation of the order $\lambda \sim 0.1 \mathrm{~nm}$. Theoretical calculations show, that self-induced amplification of spontaneous radiation on the exit of an XFEL undulator forms a pulse composed of many ultra-short peaks with duration from a fraction up to tens femtosecond. A further tailoring of the $\mathrm{X}$-ray radiation parameters is necessary for most experimental application. A quite natural solution for this task is diffraction on ideal single crystals.

A dynamical theory of diffraction of X-ray pulses with arbitrary form in the Bragg and the Laue cases was developed, which allows to consider special and temporal distribution of reflected and transmitted pulses at any given distance from a crystal with account of diffuse spreading of these pulses in the process of their propagation in space. It is shown, that super-short pulses with the duration about $\mathbf{0 . 1 - 1} \mathrm{fs}$ are strongly widened in time and are deformed in form by the diffraction process.

In the present paper I investigate the possibility of time compression of pulses, i.e. the reduction of their duration by means of the Bragg reflection. It is shown, that in the case of incident chirp pulses, for which the instantaneous frequency of radiation has a linear time dependence, and the phase - a quadratic one, it is possible to achieve for 1-10 fs incident pulse a reduction of duration by a factor of 10 . The effect is based on a large spectral width of the chirp pulses, comparable or even exceeding the typical width of a Bragg reflection for the plane wave case.

Keywords: free-electron lasers; ultrashort X-ray pulses; X-ray optics; dynamical diffraction.

PACS: 42.55.Vc

Biblography - 37 references

Received 01.12.2014

RENSIT, 2014, 6(2):177-186

DOI: 10.17725/RENSITe.0006.201412c.0177

CONTENTS

1. INTRODUCTION (177)

2. X-Ray PUlSe propagation IN fReE SpaCe (180)

3. Time Compression of X-RAY pUlses (181)

4. Conclusions (184)

AcKnowledgments (184)

References (184)

\section{INTRODUCTION}

The generation of an X-ray free-electron laser (XFEL) is based on the phenomenon of the selfamplified spontaneous emission (SASE) of highenergy electron bunches at their transmission through a lengthy undulator system [1-3].

Three XFEL projects are now actively developed: the European XFEL Facility in
Germany with radiation in hard $X$-ray range $(\lambda$ $\sim 0.1-1.6 \mathrm{~nm}$ ) [4], the $X$-ray lasers LCLS (Linac Coherent Light Source) in the USA [5] and the SCSS (Spring-8 Compact SASE Source) in Japan [6].

According to the calculations and experimental results reported in [2, 7-10], the parameters of the European XFEL and its radiation in the SASE 1 channel are expected to be as follows: electron energy $17.5 \mathrm{GeV}$, total length of superconducting undulators $\sim 150 \mathrm{~m}$, central radiation wavelength $\lambda_{0}=0.1 \mathrm{~nm}$, and the pulse full duration width at half maximum $\left(\right.$ FWHM) $\tau_{p} \sim 100 f s$. These pulses have a very irregular multispike temporal structure with a width of individual random subpulses (spikes) 
$\tau_{s} \sim 0.1-0.2 f_{s}$ and time intervals of $\sim 0.3-0.4 f_{s}$ between them, the transverse pulse size at the undulator output $r_{0} \approx 40 \mu \mathrm{m}$, and an angular divergence $\Delta \theta_{0} \approx 1 \mu \mathrm{rad}$; peak power $\approx 10 \mathrm{GW}$, average power $\approx 40 \mathrm{~W}$. The expected XFEL peak brightness should exceed that of modern third-generation synchrotron radiation sources by nine orders of magnitude [4].

$X$-ray diffraction phenomenon is widely used for monochromatization, collimation and polarization changes of $X$-rays. In this connection, great interest is the consideration of the diffraction reflection and transmission in perfect single crystals for controlling the characteristics of the laser radiation in the hard $X$-ray wavelengths, and for the development of diagnostic methods of XFEL pulse parameters.

The analysis of diffraction of XFEL radiation has been restricted so far to the approximation of a plane (unlimited) wavefront for the Bragg case "on reflection" [11-14] and for the Laue case "on transmission" $[13,15,16]$. The time structure of the incident pulse has been approximated either by a $\delta$-function $[11-13,15,16]$ or by a Gaussian $[12,14]$. Although giving some insight into the physics of short-pulse diffraction, such an approach cannot in principle take into account the presence of transverse mode structure and, even more essential, a non-uniform distribution of the field phase inside a pulse. However, such a phase distribution will inevitably arise at large, of the order of 100-1000 m, distances from the undulator to the sample or monochromator crystal. Besides, all analysis [11-16] so far has been to the reflected pulse field on the exit surface of a crystal, whereas significant practical interest is for spatial (transversal) and temporal (longitudinal) smearing of pulses during their further propagation in vacuum from an output undulator window to the crystal-monochomator or to the crystal for investigations.

In the articles [17, 18] a general theory of dynamical diffraction of $X$-ray pulses with an arbitrary spatial and temporal structure of the field on crystals with arbitrary thickness and asymmetry coefficient in the Bragg and in the Laue cases is developed. Such an approach allows us to analyze the structure of fields of forward-diffracted (transmitted) and diffracted (reflected) pulses at any distance from the crystal, and also the degree of space and time coherence of these pulses and their relation with the statistical properties of the XFEL radiation field. The analysis of pulse form and duration transformation in the process of diffraction and propagation in vacuum is also conducted. It is shown, that only the symmetrical Bragg case can be used to avoid a smearing of reflected pulses.

Time-resolved experiments, $X$-ray photon correlation spectroscopy, coherent diffraction, and phase-contrast imaging depend to a great extend on the coherent properties of $X$-ray pulses $[19,20]$. XFEL radiation is almost completely spatially coherent and is characterized by a fairly moderate temporal coherence. In the saturation mode, the length of the spatial (transverse) coherence is comparable with transverse pulse size, whereas the coherence time (longitudinal coherence) $\tau_{c}$ $\approx 0.2 f_{s}<<\tau_{p}$ much less the total duration of XFEL pulse; as a result, the relative spectral pulse width $\Delta E / E \approx 0.1 \%[8,10]$.

Diffraction reflection of $\mathrm{X}$ rays from crystals and multilayer periodic structures is widely used to make radiation monochromatic and collimated. It was shown in $[17,18]$ that, in the case of a diffraction reflection of deterministic femtosecond pulses from single crystals, the reflected pulses broaden in time by 1-2 order of magnitude, their shape significantly differs from the time profile of the incident pulse, and the peak intensity is several (or even several tenths) of a percent of the incident pulse intensity. In addition, in all cases except for the symmetric reflection in the Bragg geometry, the reflected pulse orientation changes rather nontrivially and the pulse begins to diffusely spread in space and time at distances of 0.1-1 $m$ from the crystal [18]. The reason for this is that the spectral width of 
these short incident pulses significantly exceeds the spectral range of diffraction reflection.

In $[21,22]$ based on the formalism used in the statistical optics and radio-physics [23], the statistical theory of the Bragg reflection of random femtosecond XFEL pulses from multilayer periodic structures was developed. It is shown that the use of quasi-forbidden secondorder reflections from a periodic multilayer structure $\mathrm{Al}_{2} \mathrm{O}_{3} / \mathrm{B}_{4} \mathrm{C}$ allows monochromatization of femtosecond $\mathrm{X}$-ray free-electron laser pulses at level $\Delta E / E \approx 0.04 \%$ with an efficiency of $\sim 60 \%$. The intensity, duration, and statistical characteristics of the reflected pulses are studied.

In the paper [24] we report the results of a theoretical analysis of the spatial and temporal transformations of the field of $X$-ray pulse and its statistical properties under pulse propagation in free space and at a diffraction reflection in the Bragg and Laue geometries from one crystal or two crystal oriented parallel to each other. Particular attention is paid to the influence that the pulse path length has on the spatial, temporal, and statistical pulse characteristics, because the distances from the XFEL to the first optical elements and, even more, to the measuring stations are fairly large $(400-900 \mathrm{~m})$ [48]. It has been shown that the diffraction reflection significantly increases the reflected pulse coherence time, while the shape of the temporal-coherence function of the reflected pulse differs significantly from the Gaussian incident pulse and has a characteristic triangular shape with damping oscillations at the edges. Earlier, it was investigated the influence of the spatial coherence of the $X$-ray beam, limited in space, but continuous in time, on the diffraction in crystals [25] and multilayer periodic structures [26].

As was mentioned above, the XFEL pulses are characterized by an almost complete spatial coherence and a very moderate temporal coherence, leading to a spectral width of pulses of $\Delta E / E \approx 10^{-3}$. The authors [27-29] suggested various four-chip and single-chip circuits to reduce the spectrum width to $\Delta E / E \approx 10^{-5}$, allowing us to attain a so called self-seeding mode and better laser generation with the crystal placed between two undulators. In the self-seeding regime $X$-ray pulse delayed as a result of the passage of the incident XFEL pulse on the crystal in the Bragg geometry is broadened in time and, therefore, has a narrow spectrum. It is this pulse is the seed of coherent excitation of electron bunches in the next undulator. The diffraction reflection of femtosecond pulses from single crystals and multilayer periodic structures with the aim of their monochromatization and raising the degree of temporal coherence was considered in $[17,18$, 21, 22, 24].

Pulse energies of the European FEL in channels SASE1 and SASE2, depending on the bunch charge, are $20 \div 2500 \mu \mathrm{J}$ [30], leading to average energy flows of $60 \mathrm{~W} \cdot \mathrm{cm}^{-2}$ to $80 \mathrm{~kW} \cdot \mathrm{cm}^{-2}$ in the region of the first elements of $X$-ray optics at distances of $500-900 \mathrm{~m}$ from the undulator. Allowance for and prevention of the strong thermal heating of the crystal and multilayered mirrors is one of our most serious problems.

The need to analyze the heat load, in [31] we consider the effect of such factors as the pulse energy; the temporal structure of the XFEL radiation; the distance from the undulator; the initial and maximum temperatures of the crystal; the temperature dependences of the coefficients of specific heat capacity; the heat conductivity; and the linear thermal expansion coefficient on the diffraction reflection and transmission. Spatiotemporal dependences of the distribution of the crystal temperature under the effect of pulses of a free-electron X-ray laser are found using the solution of a thermal conductivity equation. The effect of temperature, its gradient, and the deformation of the crystal lattice on the diffraction reflection and the transmission of pulses in crystals of synthetic diamond are considered.

It follows from the Van Cittert-Zernike theorem that the length of spatial coherence (LSC) of radiation $\varrho_{c}=\lambda z / \pi r_{0}$ growths as distance 
$z$ increases and the source size $r_{0}$ decreases, where $\lambda$ is the wave length [23]. A number of the channels of synchrotron radiation sources are therefore being updated to extend their length and reduce the transverse dimensions of electron bunches. For example, the channels of $X$-ray free-electron lasers and SPring- 8 and APS synchrotron sources can be as long as $1 \mathrm{~km}$ [3].

As was noted above, an expression for the length of spatial coherence $\varrho_{c}$ was obtained for a monochromatic source and a completely incoherent ( $\delta$-correlated in space) source typical of heat radiation. For the synchrotron radiation of a third-generation LSC, length $\varrho_{0}$ at the output window of the undulator can be commensurate with source size $r_{0}$ [32]; for $X$-ray free-electron lasers, $\varrho_{0}$ is often greater (and even much greater) than $r_{0}[30,32]$. The Van Cittert-Zernike theorem was extended in [21, 22, 24, 25, 32-34] to sources with arbitrary lengths of spatial coherence, with due account of the parabolic bending of the regular part of the initial wave front $[21,22,24$, 25, 34].

In the monograph [23] (p. 294), it was noted that as distance $z \geq z_{c}$ from a spatially $\delta$-correlated source grows, the partial temporal coherence of its radiation field (i.e., its nonmonochromaticity) begins to affect the spatial coherence; an estimate was offered for the corresponding critical distance $z_{c} \approx c \tau_{c}\left(\pi r_{0} / \lambda\right)^{2}$, where $\tau_{c}$ is the coherence time. This effect may be ignored at distances $z<<z_{i}$. In $[21,22,24$, $34]$, it was shown that as the length of the active channel of a free-electron $X$-ray laser grows, the temporal coherence of pulses, which was ab initio poor $\left(\tau_{c} \sim 0.2 f s\right.$; i.e., $\left.\Delta E / E \sim 10^{-3}[30]\right)$, can lower an initially high degree of spatial coherence. For the typical parameters of a freeelectron $X$-ray laser $\left(\lambda \sim 0.05-0.16 \mathrm{~nm}, r_{0} \sim 10-50\right.$ $\left.\mu m, \tau_{c} \sim 0.1-0.3 f s[30]\right)$, critical distance $z_{c} \geq 1 \mathrm{~km}$ [21, 22, 24, 34].

In [35] conducted a generalization of Van Cittert-Zernike theorem on sources with arbitrary spatial and temporal coherence. This work presents the results from a theoretical analysis of variation in the spatial coherence function versus the distance, size, length of spatial coherence, and coherence time of a radiation source. A more general expression for the critical distance is obtained; it is also shown that this distance shrinks, relative to the above simple estimate for $\mathrm{zc}$, as the length of spatial coherence of the source radiation grows, what is characteristic for X-ray free-electron laser. We also show that the form of the spatial coherence function is distinct from the original Gaussian form and depends on the choice of a point in the cross section of a beam or a pulse.

As was noted above, X-ray diffraction in crystals is an effective method of managing such characteristics of the incident radiation as monochromaticity, angular divergence, duration and shape of the reflected and transmitted pulses. In the present paper, based on the results obtained earlier in $[17,18,21,22,24]$, the possibility of temporal pulse compression, i.e. reduce their duration as a result of Bragg reflection from perfect single crystals is investigated.

It is shown, that in the case of incident chirp pulses, for which the instantaneous frequency of radiation has a linear time dependence, and the phase - a quadratic one, it is possible to achieve for 1-10 fs incident pulse a reduction of duration by a factor of 10 . The effect is based on a large spectral width of the chirp pulses, comparable or even exceeding the typical width of a Bragg reflection for the plane wave case.

\section{X-RAY PULSE PROPAGATION IN FREE SPACE}

Before proceeding to the consideration of diffraction XFEL pulses in a single crystal, we first consider the transformation of the pulse as it propagates in free space in the path from the output window of the undulator to the crystal. At exit from undulator in the plane $\varrho=(x, y)$ at $z=0$ the XFEL pulse radiation field is given by $E_{0}(\boldsymbol{\varrho}, t)=A_{0}(\boldsymbol{\varrho}, t) \exp \left(-i \omega_{0} t\right)$, 
where the amplitude $A_{0}(\boldsymbol{Q}, t)$ is a complex slowly varying function of time (wave package), $\omega_{0}$ is an average (central) frequency. A slow variation of amplitude implies, that $\left|d A_{0} / d t\right|$ $<<\omega_{0}\left|A_{0}\right|$. If $\tau_{0}$ is the characteristic time of a pulse, $\Delta \omega \approx 1 / \tau_{0}$ is the spectral width of a wave package and $\Delta \omega / \omega_{0}<<1$ (it is the condition of quasi-monochromaticity).

Let us now find the field $E(\mathbf{r}, t)$ at any point of space $\mathbf{r}=(\boldsymbol{\varrho}, z)$ and at any moment of time t. Expanding the field (1) over a plane waves provides

$$
E_{0}(\boldsymbol{\rho}, t)=\int_{-\infty}^{\infty} \int_{-\infty}^{\infty} E_{0}(\mathbf{q}, \omega) \exp (i \mathbf{q} \boldsymbol{\rho}-i \omega t) d \mathbf{q} d \omega,
$$

where spectral amplitudes $E_{0}(\mathbf{q}, \omega)$ are defined as

$$
E_{0}(\mathbf{q}, \omega)=1 /(2 \pi)^{3} \int_{-\infty}^{\infty} \int_{-\infty}^{\infty} E_{0}(\boldsymbol{\rho}, t) \exp (-i \mathbf{q} \boldsymbol{\rho}+i \omega t) d \boldsymbol{\rho} d t .
$$

Here $\mathbf{q}=\left(q_{x}, q_{x}\right)$ is a transversal vector. The field $E(\mathbf{r}, t)$ should satisfy to the wave equation in free space

$$
\Delta E-\left(1 / c^{2}\right) \partial^{2} E / \partial t^{2}=0
$$

with a boundary condition $E(\varrho, z=0, t)=E_{0}(\boldsymbol{\varrho}$, t). It is easily to show, that the required field has the following general integral form:

$$
E(\mathbf{r}, t)=\int_{-\infty}^{\infty} \int_{-\infty}^{\infty} E_{0}(\mathbf{q}, \omega) \exp \left(i \mathbf{q} \mathbf{r}+i k_{z} z-i \omega t\right) d \mathbf{q} d \omega,
$$

where $k_{z}(\mathbf{q}, \omega)=\left(k^{2}-q^{2}\right)^{1 / 2}, k=\omega / c$.

We shall obtain an expression for the field $E(\mathbf{r}$, $t$ in so called the quasi-optical approximation [36], i.e. under the assumption, that the function $E_{0}(\mathbf{q}, \omega)$ (3) significantly differs from zero only at $|\mathbf{q}|<<k$. Such approximation is justified in the case, when the characteristic size $a_{0}$ of an initial pulse is much larger than the wavelength $\lambda=2 \pi / k$. In this case it is possible to expand $k_{z}$ in a series keeping terms up to the square terms over $q$ :

$$
k_{z} \approx k_{0}+\Omega / c-q^{2} / 2 k_{0}
$$

where $k_{0}=\omega_{0} / c=2 \pi / \lambda_{0}, \Omega=\omega-\omega_{0}$. Substituting (1), (3) and $k_{z}$ into (4), we shall obtain

$$
E(\mathbf{r}, t)=A(\mathbf{r}, t) \exp \left(k_{0} z-i \omega_{0} t\right) \text {. }
$$

Here $A(\mathbf{r}, t)$ is a slowly varying complex amplitude, which has the following form:

$$
A(\mathbf{r}, t)=\int_{-\infty}^{\infty} \int_{-\infty}^{\infty} G\left(\boldsymbol{\rho}-\boldsymbol{\rho}^{\prime}, z\right) A_{0}\left(\boldsymbol{\rho}^{\prime}, t-z / c\right) d \mathbf{\rho}^{\prime},
$$

with the Green function of the free space (so called propagator)

$$
G\left(\boldsymbol{\varrho}-\boldsymbol{\varrho}^{\prime}, z\right)=\left(1 / i \lambda_{0} z\right) \exp \left[i \pi\left(\boldsymbol{\varrho}-\boldsymbol{\varrho}^{\prime}\right)^{2} / \lambda_{0} z\right] \text {. }
$$

For numerical calculations, but also for a more detailed analysis of the features of XFEL pulse diffraction it is convenient to use the following equivalent spectral representation for slowly varying amplitude:

$$
A(\mathbf{r}, t)=\int_{-\infty}^{\infty} \int_{-\infty}^{\infty} A_{0}(\mathbf{q}, \Omega) \exp \left[i \mathbf{q} \boldsymbol{\rho}-i q^{2} z / 2 k_{0}-i \Omega(t-z / c)\right] d \mathbf{q} d \Omega,(8)
$$

where $A_{0}(\mathbf{q}, \Omega)$ is the Fourier amplitude of a source field $A_{0}(\varrho, t)$ in the plane $z=0$.

From equation (6) it is clear, that in vacuum, as in medium without dispersion, the perturbation reaches an observation point $z$ after a time period $z / c$, and this delay does not depend on the wave spectral structure. In other words, the temporal structure of a pulse does not vary during the propagation in free space, whereas the space distribution undergoes diffraction induced diffusion, connected with the limited cross section of a pulse $[17,18,24]$.

We shall investigate the modification of amplitude and phase of a wave depending on the distance $z$ for a practically important example of the Gaussian pulse with a quadratic variation of the initial phase:

$$
A_{0}(\varrho, t)=\exp \left[-\left(\varrho / a_{0}\right)^{2}-\left(t / \tau_{0}\right)^{2}+i \varphi_{0}(\varrho)+i \psi_{0}(t)\right] \text {, }
$$

where $a_{0}$ is a characteristic width of a pulse in a plane $z=0, \tau_{0}$ is the duration of a pulse; $\varphi_{0}(\varrho)$ $=\beta_{e}\left(\varrho / a_{0}\right)^{2}$ and $\psi_{0}(t)=\beta_{\tau}\left(t / \tau_{0}\right)^{2}$ are spatial and temporal phases, accordingly. Here $\beta_{e}$ and $\beta_{\tau}$ are dimensionless parameters, equal to the phase at $\varrho=a_{0}$ and $t=\tau_{0}$, accordingly. In the case of a flat phase front the value $\beta_{o}=0$. Substitution of (9) into (6) or (8) results in the following exact analytical expression for the complex amplitude in the observation plane $:$

$$
A(\varrho, z, t)=|A| \exp \left[i \varphi_{z}(\varrho)+i \psi_{0}(t-z / c)\right] \text {, }
$$

where

$$
|A|=(1 / W) \exp \left[-\rho^{2} / r_{0}^{2}-(t-z / c)^{2} / \tau_{0}^{2}\right],
$$




$$
\begin{aligned}
& W=\left[\left(1+\beta_{\varrho} D\right)^{2}+D^{2}\right]^{1 / 2}, \quad r_{0}=a_{0} W, \\
& \varphi_{\tau}(\varrho)=\beta_{\tau}\left(\varrho / r_{0}\right)^{2}-\operatorname{arctg}\left[D /\left(1+\beta_{\varrho} D\right)\right], \\
& \beta_{z}=\beta_{\rho}+\left(1+\beta_{\rho}^{2}\right) D .
\end{aligned}
$$

Here $D=z / L_{d}$ is dimensionless diffraction length (the so called wave parameter [36]), and $L_{d}=\pi a_{0}^{2} / \lambda_{0}$ is the distance, at which $D=1$. If, for example, $a_{0}=30 \mu \mathrm{m}$ and $\lambda_{0} \approx 0.1 \mathrm{~nm}$, then $L_{d} \approx 30 \mathrm{~m}$.

As it is clear from (10), during the propagation of a pulse the wave front of a wave is distorted. At distances $D>>1$ the amplitude of a wave decreases as $|\mathrm{A}| \sim L_{d} / \mathrm{z}$, and the cross-section of a pulse grows according to the linear law $r_{0}(z)$ $\approx a_{0}\left(1+\beta_{e}{ }^{2}\right)^{1 / 2} z / L_{d^{*}}$ The width of angular $\left(\Delta \vartheta_{d_{d}}\right)$ and frequency spectrum $(\Delta \Omega)$ do not depend on distance $z$ and are determined by the values $\lambda_{0}, a_{0}$ and $\tau_{0}$, but also by parameters of an initial phase $\beta_{\varrho}$ and $\beta_{\tau}$ :

$$
\begin{aligned}
& \Delta \theta_{d}=\left(\lambda_{0} / \pi a_{0}\right)\left(1+\beta_{\rho}^{2}\right)^{1 / 2}, \\
& \Delta \Omega=\left(2 / \tau_{0}\right)\left(1+\beta_{\tau}^{2}\right)^{1 / 2} .
\end{aligned}
$$

The consideration of diffraction of any $X$-ray pulse with a general form (5) or in the special case (10) represents a rather difficult problem. However, if the cross-section size of a pulse in the location of the crystal $r_{0}>>\Lambda$, where $\Lambda$ is the extinction length, and the width of an angular spectrum $\Delta \vartheta_{d}<<\Delta \vartheta_{B}$, where $\Delta \vartheta_{B}$ is the width of diffraction reflection curve, the problem is much simplified. For example, if (as in the project European XFEL [30]), wavelength $\lambda_{0} \approx 0.15 \mathrm{~nm}$, pulse size $a_{0} \approx 50 \mu \mathrm{m}, \beta_{\mathrm{e}} \approx \pi / 2$ and the distance from the $X$-ray laser $z \approx 500 m(D=9.8)$, then pulse size in the region of the crystal $r_{0} \approx 0.1 \mathrm{~mm}$ and angular divergence $\Delta \vartheta_{d} \approx 0.4$ ", whereas for the reflection (220) from the single crystal Si one has extinction length $\Lambda=2.16 \mu \mathrm{m}$ and the Bragg width $\Delta \vartheta_{B}=2.56 "$. In this case it is possible to neglect the dependence of amplitude and phase of a pulse from the transverse coordinate $x$, to neglect the edge effects and to take into account only dependence of the field on the time $t$.

\section{TIME COMPRESSION OF X-RAY PULSES}

In the previous papers $[12-14,17,18]$ we saw, that in the case of incidence of a super-short $X$-ray pulse on a crystal the duration of a reflected pulse is much increased. We shall now discuss an opportunity of time compression of $X$-ray pulse, i.e. the generation of a reflected pulse with duration shorter than the incident pulse. We shall obtain also the ratio for parameters of an incident pulse and the crystal, necessary for time compression.

Let us present the field of incident plane quasi-monochromatic wave of $X$-ray pulse in a form

$E_{i n}(t)=A_{\text {in }}(t) \exp \left(-i \omega_{0} t\right)$,

where $A_{\text {in }}(t)$ is slowly varying complex amplitude. We shall consider for clarity a Gaussian pulse with quadratic modulation of the phase $\varphi(t)$ :

$A_{i n}(t)=\exp \left[-\left(t / \tau_{0}\right)^{2}+i \varphi(t)\right]$,

where time-depended phase $\varphi(t)=\beta\left(t / \tau_{0}\right)^{2}$. Here $\tau_{0}$ is the duration of a pulse, $\beta$ is the constant, which is numerically equal to a phase of a pulse field amplitude at times $\mathrm{t}= \pm \tau_{0}$. The quadratic dependence of a phase on time means linear dependence of an instantaneous frequency on time: $\omega(t)=\omega_{0}-\beta t / \tau_{0}^{2}$.

Such a pulse, in which the instantaneous frequency varies in time, is called a chirp pulse, and $\beta$ is the chirp parameter.

The wavelength of a generated XFEL radiation is determined by the expression $\lambda \approx d_{u n} / 2 \gamma^{2}$, where $d_{u n}$ is the space period of the undulator, $\gamma=E / m c^{2}$, $E$ is the energy of relativistic electron [1-3]. The chirp is generated by the loss of electron energy in an undulator $(\leq 10 \%$ [1-3]) as a result of the induced deceleration and grouping in the bunches by transfer of energy in a form of $X$-ray quanta in a field of bremsstrahlung radiation along the axis of an electronic beam.

The frequency spectrum of the incident pulse (12) has the form

$$
A_{i n}(\Omega)=\left[\tau_{0} / 2 \pi^{1 / 2}(1-i \beta)^{1 / 2}\right] \exp \left[-\Omega^{2} \tau_{0}^{2} / 4(1-i \beta)\right] .
$$


Half-width of this spectrum at the level $\mathrm{e}^{-1}$ is equal to

$$
\Delta \Omega_{\text {in }}=\left(2 / \tau_{0}\right)\left(1+\beta^{2}\right)^{1 / 2}
$$

It is clear from expression (14), that at weak phase modulation $(\beta<<1)$ the width of a spectrum is determined by the pulse duration $\tau_{0}$ : $\Delta \Omega_{\text {in }}=2 / \tau_{0}$. In the case of strong modulation, when $\beta>>1$, the width of a spectrum is increased with increasing of $|\beta|$ irrespective of a sign of parameter of modulation $\beta: \Delta \Omega_{\text {in }} \approx 2|\beta| / \tau_{0}$.

We shall consider now the Bragg reflection and transmission of an $X$-ray pulse. The spectral distribution of a reflected pulse $A_{R}(\Omega)$ is determined by the product

$$
A_{R}(\Omega)=A_{\text {in }}(\Omega) R(\Omega) \text {, }
$$

where $R(\Omega)$ is complex amplitude coefficient of the Bragg reflection of a plane wave in the space of frequencies (for further details see in $[17,18])$. The spectral width of the function $\mathrm{R}(\Omega)$ has the form [37]

$\Delta \Omega_{B}=\omega_{0} \Delta \theta_{B} \operatorname{ctg} \theta_{B}$,

where $\Delta \theta_{B}$ is the angular width of the curve of diffraction reflection. In the case of symmetric reflection from a thick crystal $(d>\Lambda$, where $\Lambda$ is the extinction length [37]) the width $\Delta \theta_{B}=\lambda / 2 \pi \Lambda \cos \theta_{\mathrm{B}}$. In the case of a thin crystal with thickness $d<<\Lambda$ (kinematical approximation) $\Delta \theta_{B}=\lambda / 2 d \cos \theta_{\mathrm{B}}$.

In Fig. 1 the frequency spectrum of an incident $X$-ray pulse is shown for various values

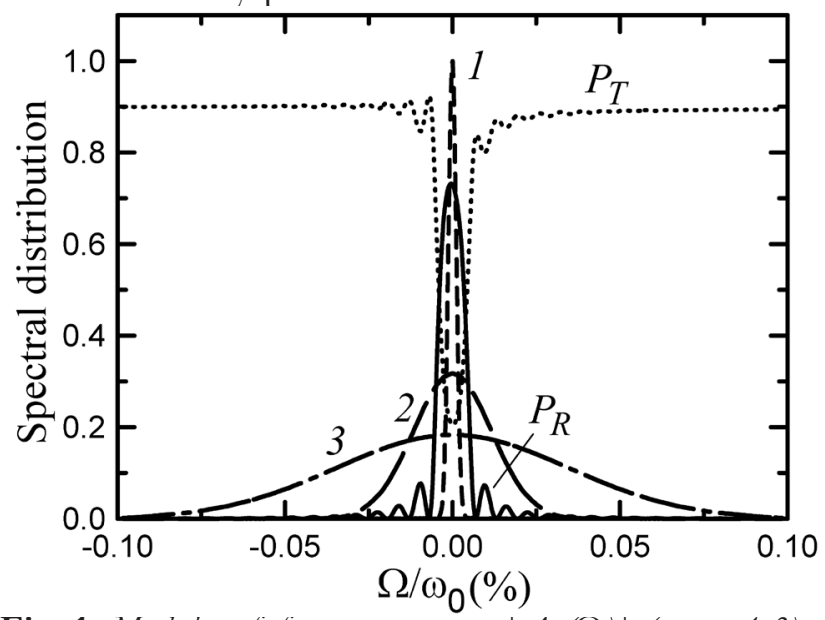

Fig. 1. Modulus of frequency spectra $\left|A_{i n}(\Omega)\right|$ (curves 1-3) of an incident $X$-ray pulse for different values of chirp parameter $\beta$ : $O$ (curve 1), 10 (curve 2), 30 (curve 3). Functions $P_{R}$ and $P_{T}$ are the intensity curves of the Bragg reflected and transmitted waves in the case of plane monochromatic incident wave, respectively. Pulse duration $\tau_{0}=10 \mathrm{fs}$, crystal thickness $d=3 \mu \mathrm{m}$, symmetric Bragg reflection Si(220), wavelength $\lambda=0.154 \mathrm{~nm}$. of chirp parameter $\beta$ together with the reflection and transmission curves.

From (15) it is follows, that the spectral width of the reflected pulse $\Delta \Omega_{R}$ is determined approximately by the expression

$\Delta \Omega_{R} \approx \Delta \Omega_{i n} \Delta \Omega_{B} /\left(\Delta \Omega_{\text {in }}^{2}+\Delta \Omega_{B}^{2}\right)^{1 / 2}$.

The duration of a reflected pulse $\tau_{R} \approx 2 / \Delta \Omega_{R}$ strongly depends on the ratio of spectral width $\Delta \Omega_{\text {in }}$ (14) and $\Delta \Omega_{B}(16)$ We shall consider two limiting cases.

a) The case of a long pulse, i.e. the pulse with a narrow spectrum: $\Delta \Omega_{\text {in }}<<\Delta \Omega_{B}$. In this case from (17) it is follows, that $\Delta \Omega_{R} \approx \Delta \Omega_{\text {in }}$ and the duration of a reflected pulse is equal to

$\tau_{\mathrm{R}} \approx \tau_{0} /\left(1+\beta^{2}\right)^{1 / 2}$.

Thus, in the case of strong phase modulation $(\beta>>1)$ a significant time compression of reflected (Fig. 2) and transmitted (Fig. 3) pulses with $\tau_{R}<<\tau_{0}$ is possible.

b) The case of a short incident pulse, i.e. the pulse with a wide spectrum: $\Delta \Omega_{\text {in }}>\Delta \Omega_{B}$. In this case from (17) follows, that $\Delta \Omega_{R} \approx \Delta \Omega_{B}$ and the duration of a reflected pulse is

$\tau_{\mathrm{R}} \approx \tau_{b} \equiv 2 / \Delta \Omega_{B}$.

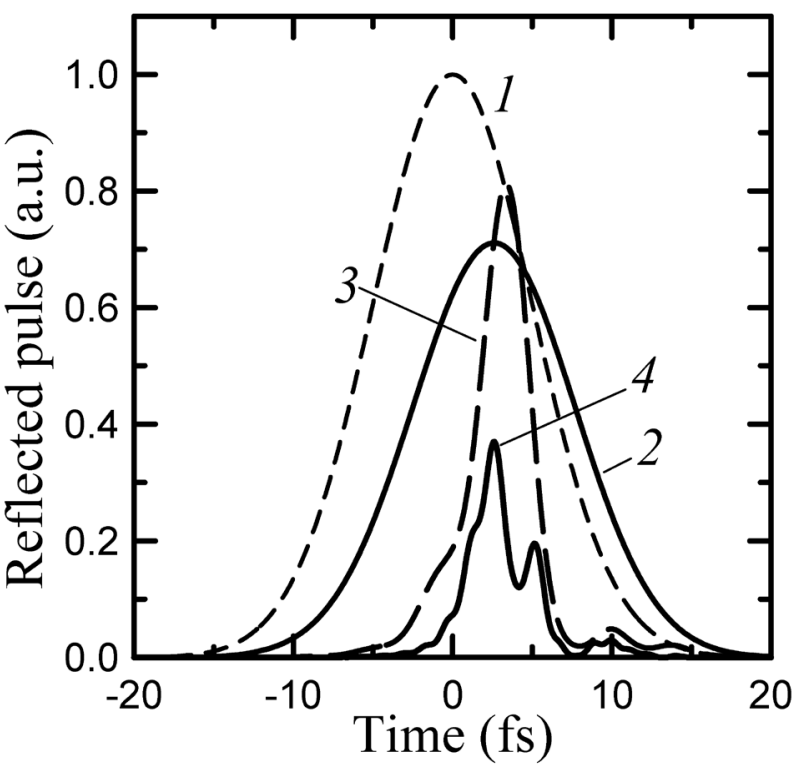

Fig. 2. Intensity of the incident pulse (curve 1) and intensity of the reflected pulse (curves 24) versus time for different values of chirp parameter $\beta$ : 0 (curve 2), 10 (curve 3), 30 (curve 4). The units of the abscissa are femtoseconds, whereas the ordinate is in arbitrary units. Time compression of a diffracted pulse by a factor of 4.4 and its splitting can be clearly seen (curve 4). Otherpulse and crystal parameters are as in Fig. 1. 


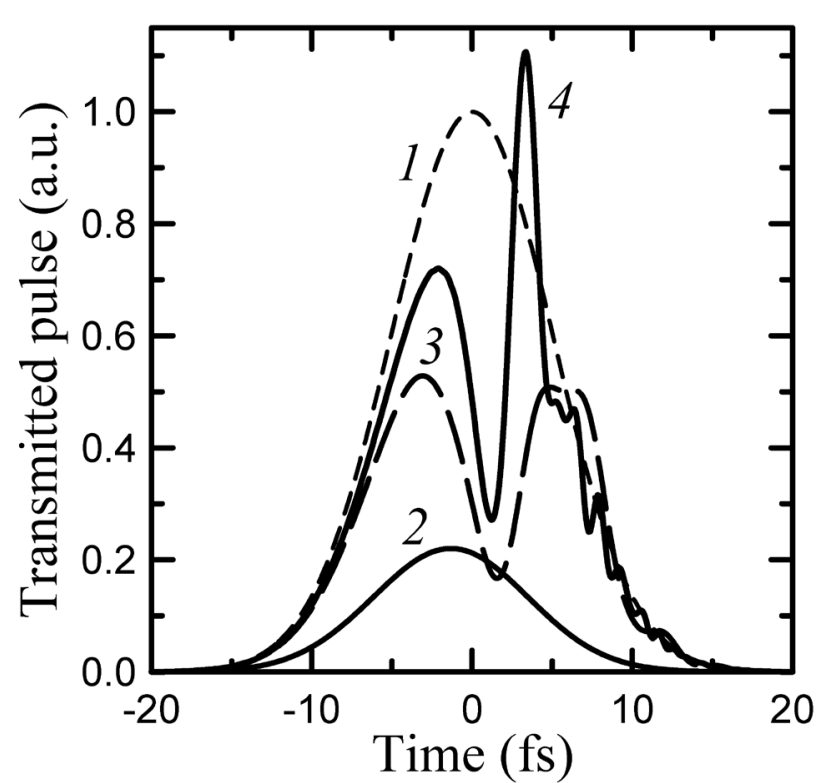

Fig. 3. Intensity of the incident pulse (curve 1) and intensity of the transmitted pulse (curves 2-4) versus time for different values of chirp parameter $\beta$ : 0 (curve 2), 10 (curve 3), 30 (curve 4). The transmitted pulse becomes higher and consists of two narrow peaks. Other pulse and crystal parameters are as in Fig. 1.

Clearly, the duration of the reflected pulse is defined by the spectral width of diffraction reflection curve alone. Thus, pulses with the duration $\tau_{0}<<\tau_{b}$ can not be compressed in principle (see Fig. 4).

In the case of a thick crystal the duration $\tau_{h}$ $=2 \Lambda \sin \theta_{B} / c$. For instance, for the symmetrical

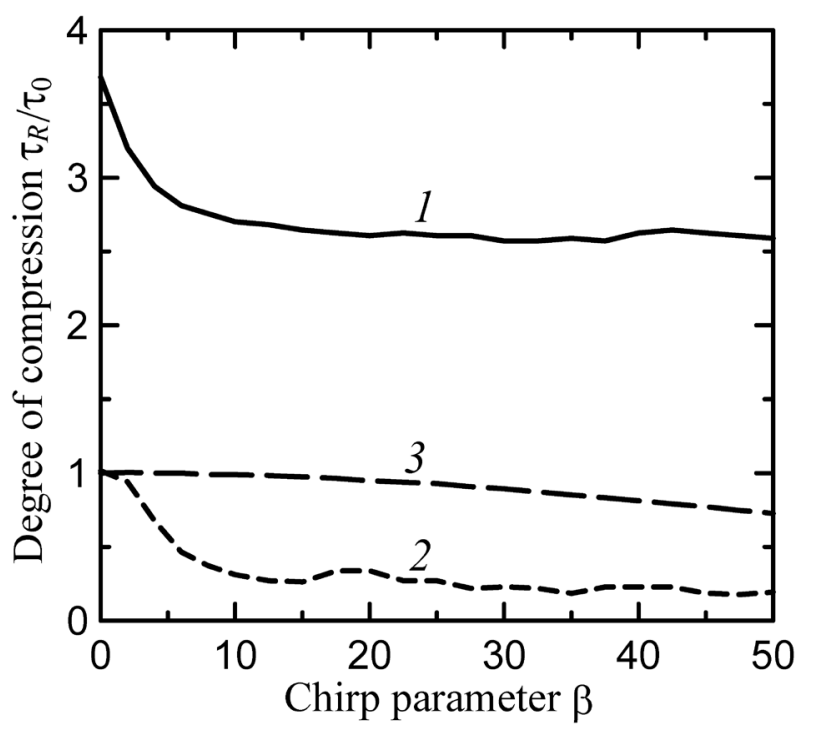

Fig. 4. Dependence of degree of time compression $\tau_{R} / \tau_{0}$ on the chirp parameter $\beta$ at different incident pulse duration $\tau_{0}: 1 f_{s}$ (curve 1), $10 \mathrm{fs}$ (curve 2), and $100 \mathrm{fs}$ (curve 3). Significant time compression is carried out only for incident X-ray pulses with the duration $\tau_{0} \sim 10 f_{s}$. Crystal thickness $d=3 \mu \mathrm{m}$, symmetric Bragg reflection Si(220), wavelength $\lambda=0.154 \mathrm{~nm}$. reflection $\operatorname{Si}(220)$ and $\lambda=0.154 \mathrm{~nm}$ the value $\tau_{b} \approx$ $5.7 f s$. The boundary value of $\tau_{b}$ can be reduced by use of a thin crystal with thickness $d<<\Lambda$, for which $\tau_{b}=2 d \sin \theta_{B} / \pi c$. If, for example, $d=0.1 \Lambda$, then $\tau_{b}=0.2 f s$. However in this case the intensity of a reflected pulse sharply decreases together with the coefficient of the Bragg reflection $|\mathrm{R}|^{2}$ $\sim(d / \Lambda)^{2}$.

For super-short pulses with $\tau_{0}<\tau_{b}$ the duration of a reflected pulse is determined, actually, by the time of propagation of a wave with speed $c$ in sub-surface layer with the thickness $\Lambda$ in the case of a thick crystal and in a crystal with thickness $d$ in the case of a thin crystal.

From Fig. $\mathbf{5}$ it is clear, that with the reduction of a crystal thickness the reflected pulse duration also decreases. Full width at halfmaximum $\Delta t_{R}=1.18,4.45,2.03$ and $1.21 f s$ for curves $1-4$, accordingly. From these data it is clear, that the condition $\tau_{0} \sim \tau_{b}$ is met only for a thin crystal with $d=0.2 \mu \mathrm{m}$ (curve 4), therefore only in this case it is possible to carry out a time compression of an incident chirp pulse (see Fig. 6).

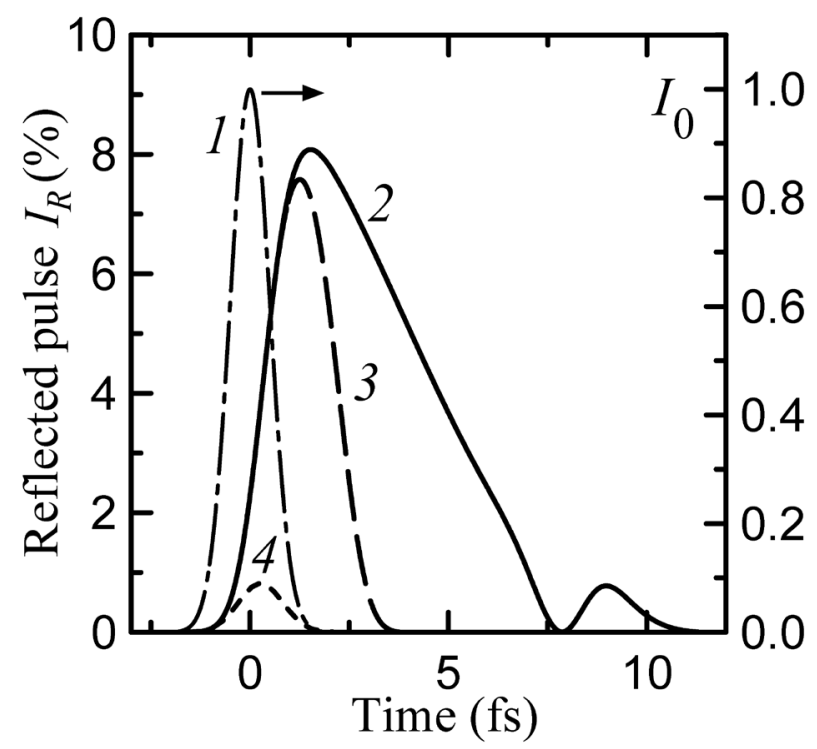

Fig. 5. Intensity of the incident pulse with the duration $\tau_{0}=1 \mathrm{fs}$ (curve 1, right ordinate scale) and the intensity of the reflected pulse (curves 24) versus time at different thickness of a crystal d: $3 \mu \mathrm{m}$ (curve 2), $1 \mu \mathrm{m}$ (curve 3), $0.2 \mu \mathrm{m}$ (curve 4). The units of the abscissa are femtoseconds, whereas the ordinate is in percents with respect to the incident pulse maximum. Symmetrical Bragg case, reflection Si(220), chirp parameter $\beta=0$, wavelength $\lambda=0.154 \mathrm{~nm}$. 


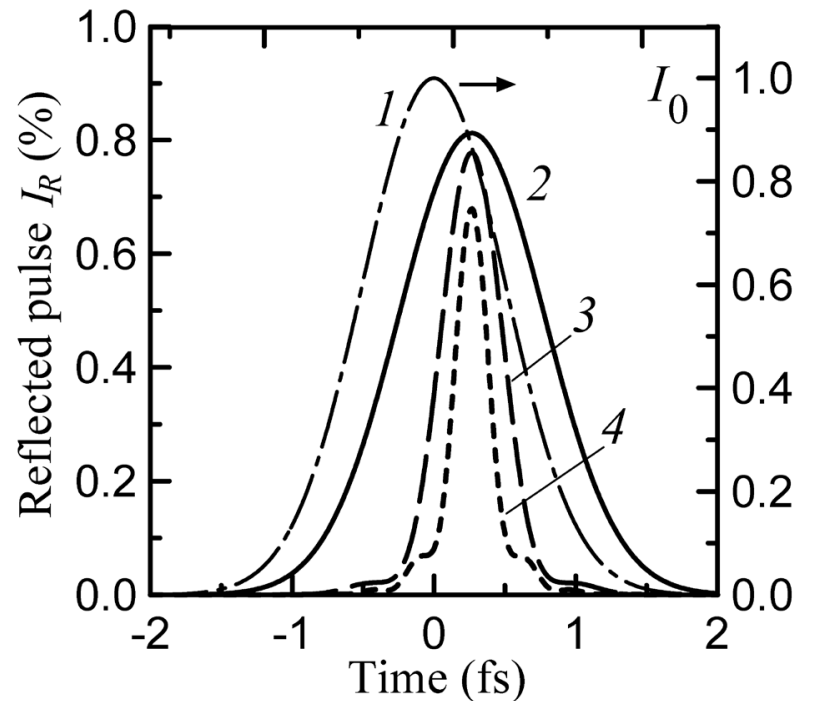

Fig. 6. Time compression of an incident pulse with the duration $\tau_{0}=1 \mathrm{fs}$ (curve 1, right ordinate scale) after the Bragg reflection (curves 2-5) from a thin crystal with the crystal thickness $d=$ $0.2 \mu \mathrm{m}$ at different values of chirp parameter $\beta$ : 0 (curve 2), 10 (curve 3), 20 (curve 4), 30 (curve 5). The units of the abscissa are femtoseconds, whereas the ordinate is in percents with respect to the incident pulse maximum. Symmetrical Bragg case, reflection Si(220), wavelength $\lambda=0.154 \mathrm{~nm}$.

With the increase of chirp parameter $\beta$ (see Fig. 6) the duration of a reflected pulse decreases almost by a factor of 6 from $\Delta t_{R}=1.21 \mathrm{fs}$ at $\beta=$ 0 (curve 2) up to $\Delta t_{\mathrm{R}}=0.21 \mathrm{fs}$ at $\beta=30$ (curve 5).

From Fig. 7 it is clear, that the reduction of thickness of a crystal also results in reduction of reflected pulse duration for a super-short incident pulse with $\tau_{0}=0.1 \mathrm{fs}$. However the

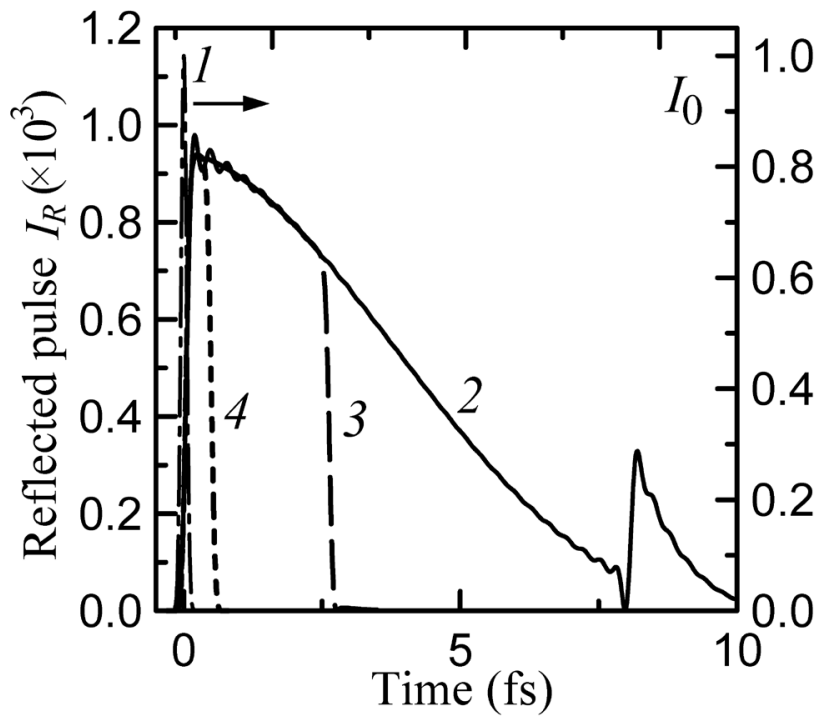

Fig. 7. Intensity of the incident pulse with the duration $\tau_{0}=0.1 \mathrm{fs}$ (curve 1, right ordinate scale), and the intensity of the reflected pulse (curves 24) versus time at different thickness of a crystal d: $3 \mu \mathrm{m}$ (curve 2), $1 \mu \mathrm{m}$ (curve 3), $0.2 \mu \mathrm{m}$ (curve 4). Symmetrical Bragg case, reflection Si(220), chirp parameter $\beta=0$, wavelength $\lambda=0.154 \mathrm{~nm}$. effect of time compression cannot be reached at any reasonable chirp parameters $\beta$, and in any practical case the duration of a reflected pulse $\Delta t_{\mathrm{R}}$ exceeds $\tau_{0}$ even for a thin crystal.

\section{CONCLUSIONS}

In conclusion, this paper presents the results of theoretical investigation of the possibility of time compression of $X$-ray free-electron laser femtoseconds pulses, i.e. the reduction of their duration under conditions of the Bragg diffraction on single crystals. It is considered the cases of short and long pulses, and also the cases of the Bragg reflections from thick and thin single crystals. It is shown, that in a case of incident chirp pulses, for which the instantaneous phase of radiation has quadratic time dependence, it is possible to achieve for 1-10 fs incident pulses a reduction of duration of the diffracted pulses by a factor of 10 .

\section{ACKNOWLEDGMENTS}

The work was supported by the Russian Foundation Base Research (Grants No. 13-0200300, No. 13-02-00760, No. 14-26-07197, No. 15-02-01502). The author is very grateful to D. Novikov for helpful discussions.

\section{REFERENCES}

1. Elton RC. X-ray lasers. Academic Press, Inc. Harcourt Brace Jovanovich, Publishers, 1990, $285 \mathrm{p}$.

2. Saldin EL, Schneidmiller EA, Yurkov MV. The physics of free electron lasers. Berlin, Springer, 1999, $484 \mathrm{p}$.

3. Fetisov GV. Sinkhrotronnoe izlucheniye. Medody issledovaniya struktury veschestva [Synchrotron radiation. Methods of the material structure investigation]. Moscow, Fizmatlit Publ., 2007, $672 \mathrm{p}$.

4. Altarelli M. (eds.), XFEL. Technical Design Report. 2006, DESY 2006-097. Hamburg, Germany, http://xfel.desy.de/tdr/index_eng. html.

5. Arthur J. LCLS Conceptual Design Report. 2002, LCLS, USA, http://www-ssrl.slac.stanford. 
$\mathrm{edu} / \mathrm{lcls} / \mathrm{cdr} /$

6. Tanaka T, Shintake T. SCSS X-FEL Conceptual Design Report, edited by Takashi Tanaka and Tsumoru Shintake. SCSS XFEL, R\&D Group, RIKEN Harima Institute/SPring-8, Japan, 2005, http://www-xfel.spring8.or.jp/ SCSSCDR.pdf.

7. Saldin EL, Scheidmiller EA, Yurkov MV. Nucl. Instr. Meth. A, 1999, 429(2):233-237.

8. Saldin EL, Schneidmiller EA, Yurkov MV. Report TESLA-FEL 2004-02, DESY, Hamburg, Germany, 2004, 39 p.

9. Saldin EL, Schneidmiller EA, Yurkov MV. New J. Phys., 2010, 12:035010(15).

10. Geloni G, Saldin E, Samoylova L, Schneidmiller E, Sinn H, Tschentscher Th, Yurkov M. New J. Phys., 2010, 12:035021(15).

11. Chukhovskii FN, Forster E. Acta Cryst. A, 1995, 51(5):668-672.

12. Shastri SD, Zambianchi P, Mills DM. J. Synchrotron Radiat., 2001, 8(7):1131-1135.

13. Shastri SD, Zambianchi P, Mills DM. Proc. SPIE, 2001, 4143:69-77.

14. Graeff W. J. Synchrotron Radiat., 2004, 11(3):261265.

15. Graeff W. J. Synchrotron Radiat., 2002, 9(1):8287.

16. Malgrange C, Graeff W. J. Synchrotron Radiat., 2003, 10(3):248-254.

17. Bushuev VA. Bull. Russ. Acad. Sci. Phys., 2005, 69(12):1903-1908.

18. Bushuev VA. J. Synchrotron Radiat., 2008, 15(5):495-505.

19. Vartanyants IA, Robinson IK. Optics Commun., 2003, 222(1-6):29-50.

20. Vartanyants IA, Robinson IK, McNulty I, David C, Wochner P, Tschentscher Th. J. Synchrotron Radiat., 2007, 14(6):453-470.

21. Bushuev V, Samoylova L. Nucl. Instr. Meth. A, 2011, 635(4):S19-S23.

22. Bushuev VA, Samoylova L. Bull. Russ. Acad. Sci. Phys., 2012, 76(2):153-158.

23. Akhmanov SA, D'yakov SA, Chirkin AS. $\checkmark$ vedeniye $v$ statisticheskuyu raiofiziku $i$ otpiku Introduction to statistical radio-physics and optics]. Moscow, Nauka Publ., 1981, 640 p.

24. Bushuev VA, Samoylova L. Cryst. Rep., 2011, 56(5):819-827.

25. Bushuev VA. Bull. Russ. Acad. Sci. Phys., 2009, 73(1):52-56.

26. Bushuev VA. Bull. Russ. Acad. Sci. Phys., 2010, 74(1):41-45.

27. Saldin E, Schneidmiller E, Shvyd'ko Yu, Yurkov M. Nucl. Instrum. Methods. A, 2001, 475(2):357362.

28. Geloni G, Kocharyan V, Saldin E. Report DESY 10-053, Hamburg, Germany, 2010, 053:1-28.

29. Geloni G, Kocharyan V, Saldin E. Report DESY 11-224, Hamburg, Germany, 2011. 224:1-13.

30. Tschentscher Th. XFEL.EU TN-2011-001, 2011, 001:1-21.

31. Bushuev VA. Bull. Russ. Acad. Sci. Phys., 2013, $77(1): 15-20$.

32. Vartanyants IA, Singer A. New J. Physics, 2010, 12:035004(23).

33. Cerbino R. Phys. Rev A, 2007, 75(5):053815(4).

34. Bushuev V, Samoylova L, Proc. SPIE, 2011, 8141: 81410T(14); doi:10.1117/12.893054.

35. Bushuev VA. Bull. Russ. Acad. Sci. Phys., 2014, 78(12):1382-1387.

36. Vinogradova MB, Rudenko OV, Sukhorukov A.P. Teoriya voln [The theory of waves]. Moscow, Nauka Publ., 1990, 432 p.

37. Pinsker ZG. Dynamical scattering of X-rays in crystals. Springer Series in Solid-State Physics, Vol. 3. Springer Verlag, Berlin. 1978, 347 p. 